\title{
A Novel Approach for Realization of Higher Order Filter using Bipolar and MOS Current-Mirrors
}

\author{
Ashish Gupta ${ }^{1,}$ Manveen S. Chadha ${ }^{2,}$ Amendra Bhandari ${ }^{3}$ Agha A. Husain ${ }^{4}$ \\ 1, 2,3,4 (Department of Electronics and Communication Engineering, I.T.S Engineering College, Plot No: 46, \\ Knowledge Park-III, Greater Noida, (U.P.), INDIA)
}

\begin{abstract}
In this paper, we have presented an approach to realize higher order current-mode filters using cascade technique of lower order filters realized using CMOS current-mirrors. This paper presents the realization of higher-order low-pass $(L P)$ active current-mode filters using cascade technique of second and third order filters. Interest in current-mode (CM) filters has been growing due to the fact that current-mode devices have wider dynamic range, improved linearity, and extended bandwidth as compared to their voltagemode counterparts. In the sub-micron era of semi-conductor technology, the minute structure of devices causes a high electric field and thus the power supply voltage is limited to a certain low value to protect the device from destruction. Current-mode signal processing is quite attractive for operation at high frequencies and low power supply voltage operation from the viewpoint of sub-micron era. The realized filters do not contain any resistors, therefore simplifying the pattern of integrated circuits and thus reducing the chip area and the cost. The proposed circuits presented in the paper using bipolar technology and CMOS technology were tested in

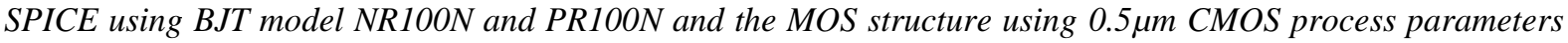
provided by MOSIS (AGILENT) and the results thus obtained were in accordance with the theoretical values.

Keywords: Continuous-time current-mode filters, Current Adders and Integrators, Bipolar and CMOS Current-Mirrors, Higher Order Filters, Cascade realization.
\end{abstract}

\section{Introduction}

Recent advancement in the field of integrated circuit technologies have miniaturized the devices used in an IC causing high internal electric field to develop. Therefore, the power supply voltage of the circuits must be reduced extremely to low value. In addition to this, the use of portable equipments has restricted the operation to a single battery [1], [2]. Also operation at high frequencies is a key desirable feature for these devices. In this respect, realizations of low-voltage and high-frequency active filters are an attractive choice in the field of signal processing.

Active filters are the best choice because they have the possibilities of integration and they are suitable for high frequency and low voltage operation. Among the numerous techniques available for the realization of active RC filters, we present two different methods in this paper namely (i) Cascade realization and (ii) Leapfrog realization [3]. These techniques of filter realizations are quite popular and can generate any type of transfer function. Traditionally, such circuits work in the continuous-time domain and have being designed as resistively terminated lossless LC filters where resonance could be employed to achieve complex poles and the desired steep transition region between the pass bands and the stop bands. With the growing pressure towards micro-miniaturization, inductors were found to be too bulky so that designers started to replace passive RLC filters by their active RC counterpart circuits where gain was obtained from operational amplifiers, and with the presence of resistors and capacitors in feedback networks, complex poles can be achieved. Active filters designed using the ubiquitous op-amp have found wide acceptance throughout the literature over the past and their mature development and technology guarantee their continued popularity in many applications.

One disadvantage of these op-amp based active RC filters is the limited frequency range of operation over which these circuits can be used: the finite bandwidth of op-amps usually confined the application to be below $100 \mathrm{KHz}$ with performance becoming deteriorating with increasing frequency [4], [5]. For operation in $\mathrm{MHz}$ range the main design challenges for the analog filters are: (i) Reliable high-frequency performance. (ii) Automatic on-chip tuning against fabrication tolerances and (iii) changing operating conditions [6] and [7].

For application as continuous-time filters, the transconductance [8], [9] should satisfy the following properties: (i) Circuits must be simple, linear and should have a wider frequency response. (ii) Must have large input and output impedances to prevent undesirable interactions and simplify circuit design. (iii) They should preferably work with low-voltage power supplies to conserve power and to be compatible with the prevalent digital technologies on the same chip. (iv) Their transconductance parameter must depend on some dc bias voltage or current to facilitate electronic tuning against environmental or processing variations [10].

In many useful active simulation of filters, we may even insist that all the transconductors are identical and all the capacitors are grounded for specially simple IC layout and processing, and implementation of 
integrated analog filters based on analog gate arrays appears to be a distinct possibility. In addition, the wider useful bandwidth of transconductances coupled with the reduced effects of circuit and device parasitics on filter performance result in far higher operating frequencies at which the circuits can function.

In the stop-band the gain of the filter changes at the rate of $20 \mathrm{db} / \mathrm{decade}$ for first-order filter which implies that for $\mathrm{n}^{\text {th }}$-order filter the gain changes at the rate of $20 \mathrm{n} \mathrm{db} / \mathrm{dec}$ de. The simplest way to build a fifthorder low-pass filter is by cascading a second order filter with a third-order section. Although there is no limit to the order of the filter that can be formed, as the order of the filter increases, so does its size. Also the accuracy declines, in that the difference between the actual stop-band response and the theoretical stop-band response increases with an increase in the order of the filter. Generally, the minimum-order filter required depends on the application specifications. Although a high-order filter necessarily provides a better stop-band response, the higher-order filters are more complex, occupy more space and are more expensive. Higher order filters provide greater roll-off rates between pass-band and stop-band, and can be necessary to achieve the required levels of stop-band attenuation or sharpness of cutoff frequency. They are more complex, requiring more components when implemented as analogue filters, or more processing when implemented digitally. The filter design process can be described as an optimization problem where each requirement contributes with a term to an error function which should be minimized. Typical requirements which are considered in the design process are: the filter should have a specific frequency response, a specific phase shift or group delay, a specific impulse response, causal, stable, localized, the computational complexity of the filter should be low and the filter should be implemented in particular hardware or software. Another important parameter of great concern is the required frequency response of the filter. In particular, the steepness and complexity of the response curve is a deciding factor for the filter order and feasibility. For many purposes, this is not sufficient. To achieve steeper slopes, higher order filters are required.

\section{Cascade Design}

This approach is based on the factorization of a transfer function $\mathrm{H}(\mathrm{s})$ into the product of lower-order terms. If the order $\mathrm{n}$ is even, the decomposition consists of $\mathrm{n} / 2$ second order terms.

$$
\mathrm{H}(\mathrm{s})=\mathrm{H}_{1}(\mathrm{~s}) \mathrm{X} \mathrm{H}_{1}(\mathrm{~s}) \mathrm{X}---\mathrm{X} \mathrm{H}_{\mathrm{n} / 2}(\mathrm{~s})
$$

If $\mathrm{n}$ is odd, the factorization includes also a first order term. Sometimes this term is combined with one of the second-order terms to create a third-order filter stage. The first-order term, if any, can be implemented with a simple RC or CR network, so all we need to know is its required cut-off frequency $\omega_{\mathrm{o}}$. The second-order terms can be implemented with any of the filters of second-order. For each of these stages we need to know $\omega_{0}$ and $\mathrm{Q}$, and $\omega_{\mathrm{z}}$ if the stage is a notch stage. The cascade approach offers a number of advantages. The design of these sections is relatively simple, and the component count is usually low. The low-output impedance of the individual sections eliminates inter-stage loading, so each section can be regarded as isolated from the others and can be tuned independently, if needed. The inherent modularity of this approach is also attractive from the economic stand point, since one can use a few standardized blocks to design a variety of more complex filters.

Mathematically, the order in which the various sections are cascaded in series is irrelevant. In practice, to avoid loss of dynamic range and filter accuracy due to possible signal clipping in the high-Q sections, the sections are cascaded in order of ascending Q's, with the low-Q stages first in the signal path. This ordering, however, does not take into account internal noise, which may be of concern in the high-Q stages, where any noise component falling under the resonance peak may be amplified significantly. So, to minimize noise, high-Q stages should go first in the cascade. In general, the optimum ordering depends on the input spectrum, the filter type and the noise characteristics of its components.

\section{Proposed Circuits}

Current-mode signal processing is quite attractive for low power supply voltage operation and high frequency application. In this paper design of current-mode filters using nMOS transistor current mirrors and pMOS transistor current sources as an active load, as reported in [4], which is a counterpart of the MOS realization [6], [7] are presented. In such designs, pMOS transistors were only used for DC current sources which provide bias currents to the current mirrors and also behave as an active load for the current mirror.

The currents of these sources must match with each other and also match with the DC currents of each current mirror to give a proper DC bias to transistors. However the matching is sometimes difficult due to the parameter mismatch between nMOS and pMOS transistors [7], [8]. Here, we present design of CMOS continuous-time filters using nMOS and pMOS transistor current mirrors. Using pMOS transistors as signal processing elements, the circuits can be simplified and problems resulting from difficulty of DC biasing can be avoided. 
Using integrators and adders, any kind of transfer function can be realized; therefore this method can be easily applied for realizing wide variety of filters. Generally, monolithic integrated filters have a difficulty on setting the filter frequency due to absolute value error of elements. In these filters, the tuning of the filter frequency can be easily achieved by adjusting the current of a single DC current source.

2.1 Realization of CMOS Current-Mode Fifth-Order Low Pass Active Filter using Cascade Realization Technique:

Fig. 1 shows the practical realization of the proposed fifth-order low pass filter where the current-mode second-order section of Fig. 2 [11] and the current-mode third-order section of Fig. 2 [12] are cascaded together to obtain the proposed circuit of a fifth-order filter.

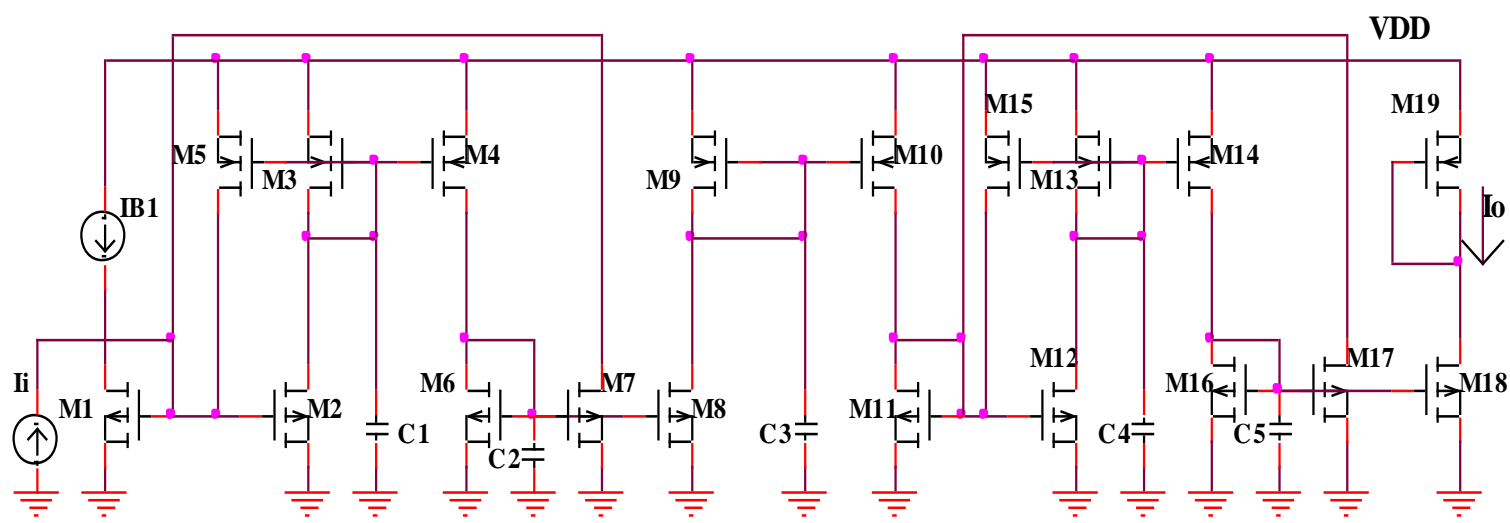

Fig. 1: Cascaded Current Mode Fifth-Order Section using CMOS Realization

Assuming that all transistors of Fig. 2 have an equal aspect ratio's that is, all the current mirrors have same $(\mathrm{W} / \mathrm{L})$ ratios, so we obtain the following transfer function:

$T_{L P}(s)=\left[\frac{1}{s^{3} C_{3} C_{4} C_{5} R^{3}+s^{2} C_{3} R^{2}\left(C_{4}+C_{5}\right)+s R\left(C_{3}+C_{5}\right)+1}\right]\left[\frac{1}{s^{2} C_{1} C_{2} R^{2}+s C_{1} R+1}\right]$

Since the DC bias currents of all transistors are equal to the bias current $\mathrm{I}_{\mathrm{B}}$, the resistances are given by,

$R_{1}=R_{2}=R_{3}=R_{4}=R_{5} \cong \frac{k T}{q} \frac{1}{I_{B 1}} \cong \frac{0.0259}{I_{B 1}}$

Equation-2.2 shows that $I_{B}$ controls $R_{1}, R_{2}, R_{3}, R_{4}$ and $R_{5}$ simultaneously and the simultaneous control of $R_{1}, R_{2} R_{3}, R_{4}$ and $R_{5}$ do not affect the $Q$ factor. Thus we can control the cut-off frequency of the filter $\omega_{0}$ without changing the $\mathrm{Q}$ factor.

\subsection{Realization of Current-Mode Fifth-Order Low Pass Active Filter using Bipolar Realization:}

Fig. 2 shows the practical realization of the proposed fifth-order low pass filter using bipolar technology where we have used the cascaded realization of the current-mode second-order and the current-mode third-order section to obtain the circuit of a fifth-order filter.

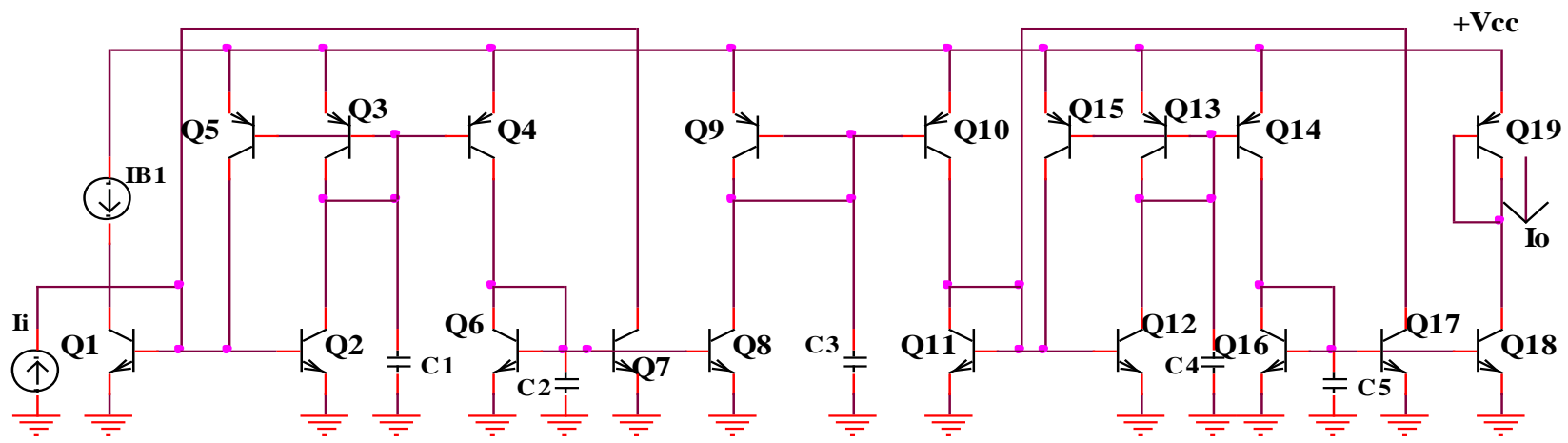

Fig. 2: Cascaded Current Mode Fifth-Order Section using Bipolar Realization 


\section{Simulation Results}

The workability of the proposed circuits were tested and verified in SPICE using $0.5 \mu \mathrm{m}$ CMOS process parameters provided by MOSIS (AGILENT) and the Bipolar parameters for NR100N and PR100N as listed in Table-1.

TABLE-1: CMOS AND BIPOLAR PROCESS PARAMETERS

\begin{tabular}{|c|c|}
\hline TRANSISTOR & PROCESS PARAMETERS \\
\hline nMOS & 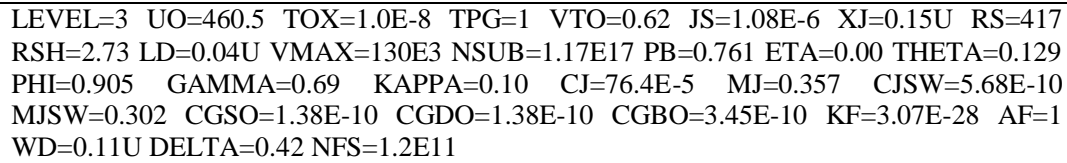 \\
\hline pMOS & 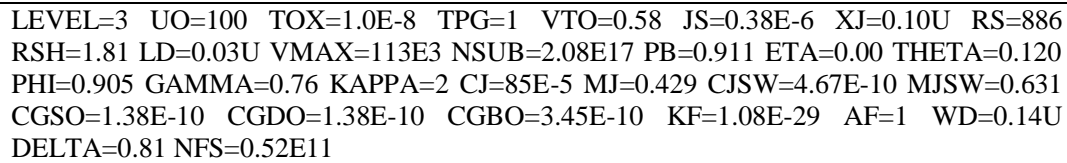 \\
\hline NR100N & $\begin{array}{l}\mathrm{IS}=121 \mathrm{E}-18 \mathrm{BF}=137.5 \mathrm{VAF}=159.4 \mathrm{IKF}=6.974 \mathrm{E}-3 \mathrm{ISE}=36 \mathrm{E}-16 \mathrm{NE}=1.713 \mathrm{BR}= \\
0.7258 \mathrm{VAR}=10.73 \mathrm{IKR}=2.198 \mathrm{E}-3 \mathrm{RE}=1 \mathrm{RB}=524.6 \mathrm{RBM}=25 \mathrm{RC}=50 \mathrm{CJE}=0.214 \mathrm{E}- \\
12 \mathrm{VJE}=0.5 \mathrm{MJE}=0.28 \mathrm{CJC}=0.983 \mathrm{E}-13 \mathrm{VJC}=0.5 \mathrm{MJC}=0.3 \mathrm{XCJC}=0.034 \mathrm{CJS}= \\
0.913 \mathrm{E}-12 \mathrm{VJS}=0.64 \mathrm{MJS}=0.4 \mathrm{FC}=0.5 \mathrm{TF}=0.425 \mathrm{E}-8 \mathrm{TR}=0.5 \mathrm{E}-8 \mathrm{EG}=1.206 \mathrm{XTB}= \\
1.538 \mathrm{XTI}=2.0\end{array}$ \\
\hline PR100N & $\begin{array}{l}\mathrm{IS}=73.5 \mathrm{E}-18 \mathrm{BF}=110 \mathrm{VAF}=51.8 \mathrm{IKF}=2.359 \mathrm{E}-3 \mathrm{ISE}=25.1 \mathrm{E}-16 \mathrm{NE}=1.650 \mathrm{BR}= \\
0.4745 \mathrm{VAR}=9.96 \mathrm{IKR}=6.478 \mathrm{E}-3 \mathrm{RE}=3 \mathrm{RB}=327 \mathrm{RBM}=24.55 \mathrm{RC}=50 \mathrm{CJE}=0.18 \mathrm{E}- \\
12 \mathrm{VJE}=0.5 \mathrm{MJE}=0.28 \mathrm{CJC}=0.164 \mathrm{E}-12 \mathrm{VJC}=0.8 \mathrm{MJC}=0.4 \mathrm{XCJC}=0.037 \mathrm{CJS}= \\
1.03 \mathrm{E}-12 \mathrm{VJS}=0.55 \mathrm{MJS}=0.35 \mathrm{FC}=0.5 \mathrm{TF}=0.610 \mathrm{E}-9 \mathrm{TR}=0.610 \mathrm{E}-8 \mathrm{EG}=1.206 \mathrm{XTB}= \\
1.866 \mathrm{XTI}=1.7\end{array}$ \\
\hline
\end{tabular}

4.1 Simulation Results for the CMOS Current-Mode Fifth-Order Low Pass Filter using Cascade Realization Technique:

For the circuit shown in Fig. 1 the ac analysis were carried out with the value of dc bias current $\mathrm{I}_{\mathrm{B}}=$ $2 \mu \mathrm{A}, \mathrm{C}_{1}=\mathrm{C}_{3}=\mathrm{C}_{5}=0.5 \mathrm{pF}, \mathrm{C}_{2}=\mathrm{C}_{4}=1 \mathrm{pF},(\mathrm{W} / \mathrm{L})_{\mathrm{P}}$ ratio $=1 \mu \mathrm{m} / 1 \mu \mathrm{m}, \quad(\mathrm{W} / \mathrm{L})_{\mathrm{N}}$ ratio $=1 \mu \mathrm{m} / 1 \mu \mathrm{m}$ and supply voltage $\mathrm{V}_{\mathrm{DD}}=1.7 \mathrm{~V}$. The value of cut-off frequency is found to be $\mathrm{f}_{\mathrm{O}}=4.0536 \mathrm{MHz}$ which is very well in agreement with the calculated theoretical value of $f_{O}=4.05 \mathrm{MHz}$. SPICE simulation results are shown in Fig. 3 .

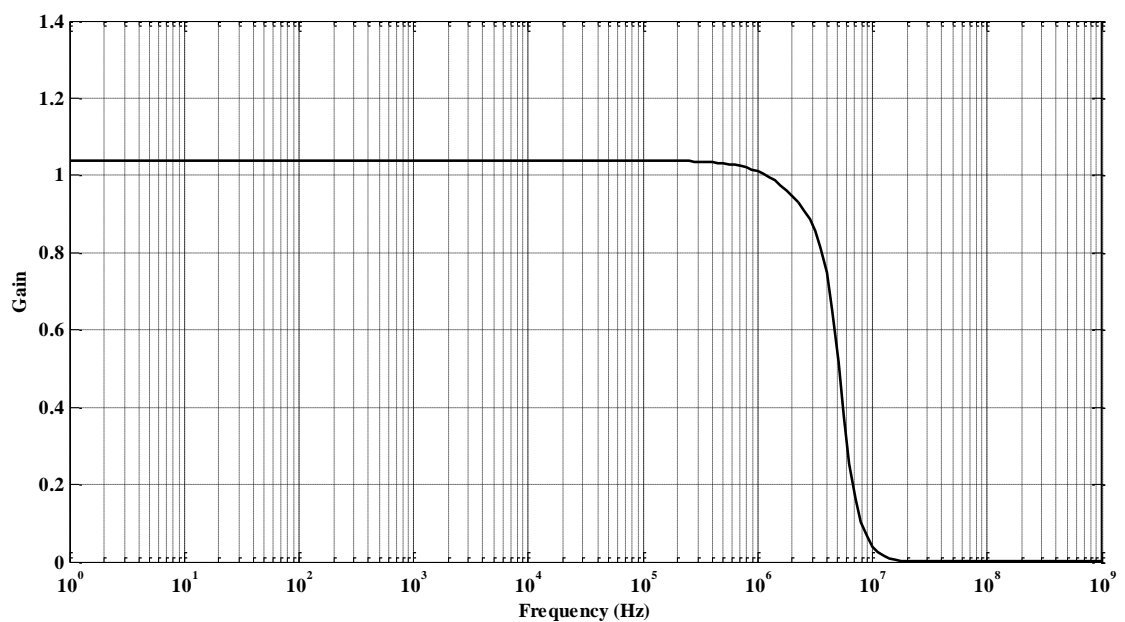

Fig. 3: Low Pass Response of Current-Mode Fifth Order Section using CMOS technology

Fig. 4 shows the change in the value of cut-off frequency with respect to the change in the values of the capacitor $\mathrm{C}_{1}$ from $0.5 \mathrm{pF}$ to $75 \mathrm{pF}$ keeping the value of capacitors $\mathrm{C}_{2}$ and $\mathrm{C}_{4}$ fixed at $1 \mathrm{pF}$ and capacitors $\mathrm{C}_{1}$ and $\mathrm{C}_{3}$ are kept fixed at $0.5 \mathrm{pF}$. The value of cut-off frequency changes from $34.28 \mathrm{KHz}$ to $4.0536 \mathrm{MHz}$ which shows that cut-off frequency varies inversely with capacitance $\mathrm{C}_{1}$. 


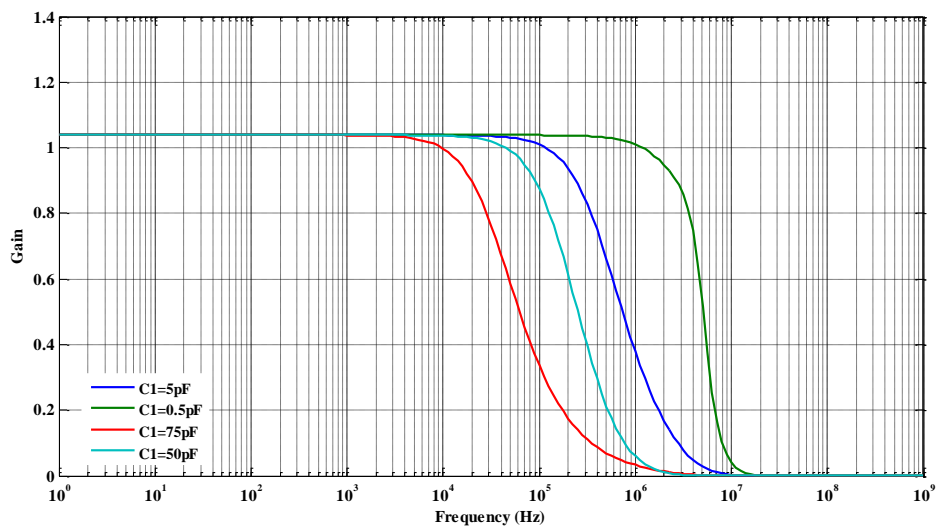

Fig. 4: Variation in the cut-off frequency with change in Capacitor value

Fig. 5 shows the change in the value of cut-off frequency with change in the value of bias current when varied from $10 \mu \mathrm{A}$ to $80 \mu \mathrm{A}$. Due to this variation the cut-off frequency varies from $4.0858 \mathrm{MHz}$ to 235.612 $\mathrm{KHz}$, thus cut-off frequency varies inversely with bias current.

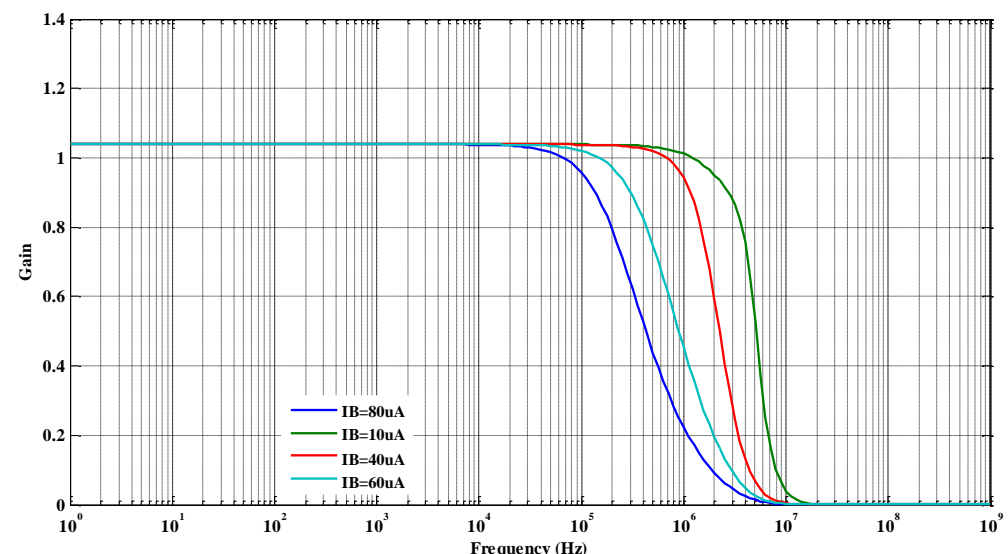

Fig. 5: Variation in the Gain with change in bias current $I_{B}$

Fig. 6 shows the change in the value of cut-off frequency with respect to the change in the values of the capacitor $\mathrm{C}_{2}$ and $\mathrm{C}_{4}$ from $5 \mathrm{pF}$ to $75 \mathrm{pF}$ keeping the value of capacitors $\mathrm{C}_{1}, \mathrm{C}_{3}$ and $\mathrm{C}_{5}$ fixed at $0.5 \mathrm{pF}$. The value of cut-off frequency changes from $21.419 \mathrm{KHz}$ to $358.783 \mathrm{KHz}$ which shows that cut-off frequency varies inversely with capacitance $\mathrm{C}_{2}$ and $\mathrm{C}_{4}$.

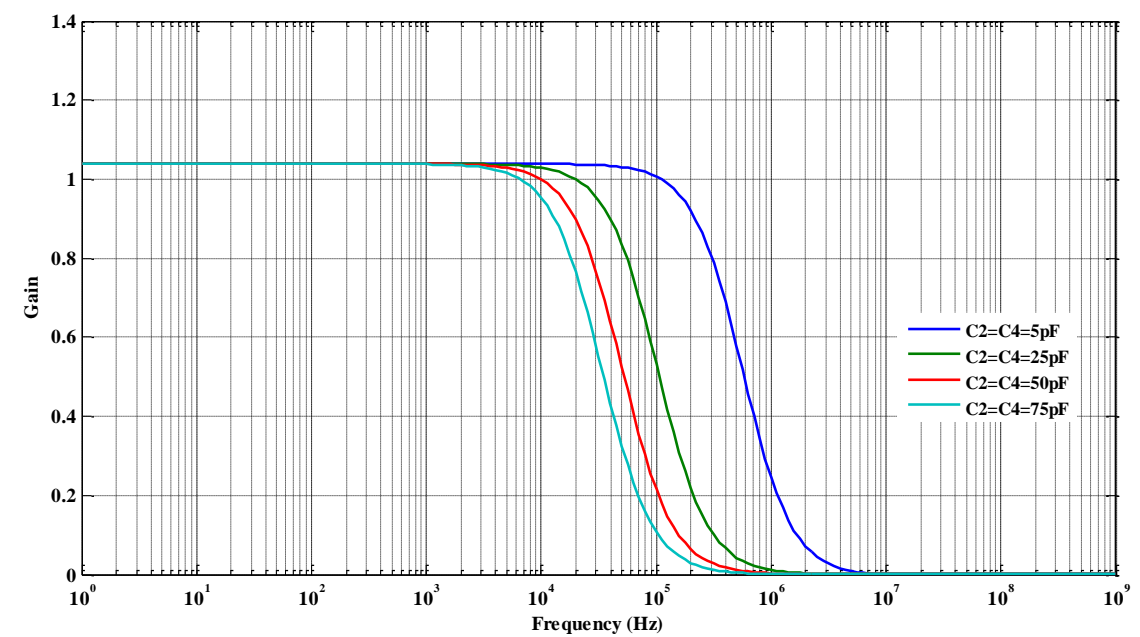

Fig. 6: Variation in the cut-off frequency with change in Capacitor values 


\subsection{Simulation Results for the Current-Mode Fifth-Order Low Pass Filter using Bipolar Technology:}

For the circuit shown in Fig. 2 the ac analysis were carried out with the value of dc bias current $\mathrm{I}_{\mathrm{B}}=$ $2 \mu \mathrm{A}, \mathrm{C}_{1}=\mathrm{C}_{3}=\mathrm{C}_{5}=0.5 \mathrm{pF}$ and $\mathrm{C}_{2}=\mathrm{C}_{4}=5 \mathrm{pF}$ and supply voltage $\mathrm{V}_{\mathrm{DD}}=1.7 \mathrm{~V}$. The value of cut-off frequency is found to be $f_{O}=1.1526 \mathrm{MHz}$ which is very well in agreement with the calculated theoretical value of $f_{O}=$ 1.16 MHz. SPICE simulation results are shown in Fig. 7.

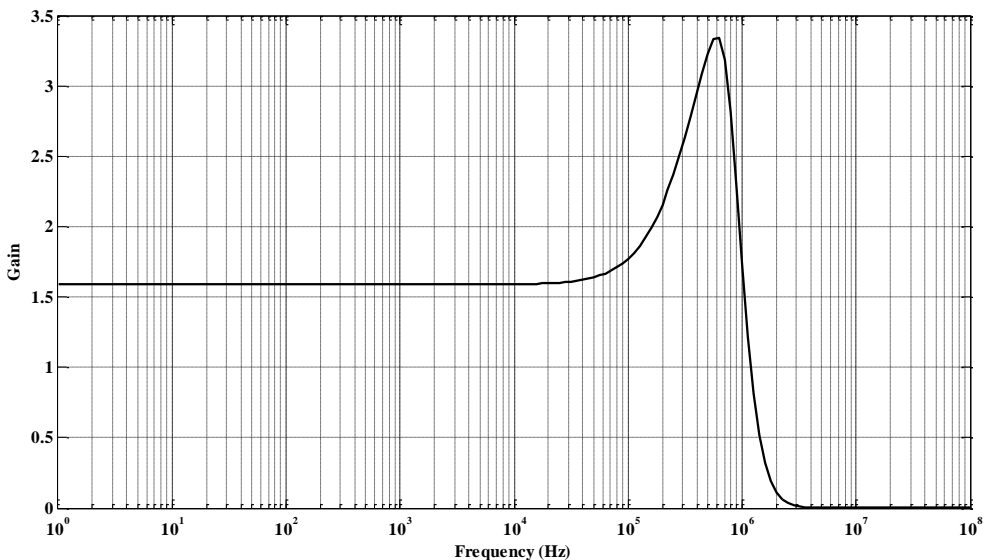

Fig. 7: Low Pass Response of Fifth-Order Current-Mode Filter using bipolar technology

\section{Conclusion}

In the given paper high frequency fifth order current-mode filter have been presented which are quite suitable for the realization in high frequencies till $10 \mathrm{MHz}$ and these filters can operate at a voltage as low as $2 \mathrm{~V}$ or less. Also, it has been verified that variations in the value of either the capacitor or the bias current improves the gain as well as the operating frequency of the filters. The frequency of this filter can be easily and widely controlled by a single DC bias current and thus provides good tunability. All the circuits were tested using SPICE and the verified results confirms the theoretical values.

\section{References}

[1] G. S. Moschytz, Low-Sensitivity, Low-Power, Active-RC Allpole Filters Using Impedance Tapering, IEEE Trans. On Circuits and Systems, CAS-46(08), 1999, 1009-1026.

[2] W. Tangsriat and W. Surakampontorn, Realization of Leapfrog Filters Using Current Differential Buffered Amplifiers, IEICE Trans. Fundamentals, E86-A, 2003, 318-326.

[3] G. H. Wang, K. Watanabe, and R. Fukui, An extended dual transformation approach to current-mode circuit synthesis, IEEE Proc. ISCAS, 1990, 2294-2295.

[4] J. C. Ahn, and N. Fujii, Current-mode continuous-time filters using complementary current mirror pairs, IEICE Trans Fundamentals, E79-A(2), 1996, 168-175.

[5] R. J. Angulo, M. Robinson, and E. S. Sinencio, Current-mode continuous-time filters: two design approaches, IEEE Tran. On Circuits and Systems, 39(5), 1992, 337-341.

[6] S. S. Lee, R. H. Zele, D. J. Allstot, and G. Liang, A continuous-time current-mode integrator, IEEE Trans. On Circuits and Systems, 39, 1991, 1236-1238.

[7] S. S. Lee, R. H. Zele, D. J. Allstot, and G. Liang, CMOS continuous-time current-mode filters for high frequency applications, IEEE J. Solid State Circuits, 28(3), 1993, 323-329.

[8] R. J. Angulo, and E. S. Sinencio, Active compensation of operational transconductance amplifier using partial positive feedback, IEEE J. of Solid-state Circuits, 25, 1990, 1024-1028.

[9] C. Toumazou, and F. J. Lidgey, Novel bipolar differential input/output current-controlled current source, Electronic Letters, 21, 1985, 199-200.

[10] B. Wilson, Analogue current-mode circuits, International Journal of Electronics Engineering Education, 26, 1989, 206-233

[11] Agha A. Husain, Manveen S. Chadha, Ashish Gupta and Amendra Bhandari, Design of High Frequency Current-Mode ContinuousTime Filter using CMOS Current-Mirrors, IOSR Journal of VLSI and Signal Processing, 3(6), 2013, 58-62.

[12] Manveen S. Chadha, Ashish Gupta, Amendra Bhandari and Agha A. Husain, Third-Order Current-Mode Filter Realization using CMOS Current-Mirror, IOSR Journal of Engineering, 3(12), 2013, 32-39.

[13] Amendra Bhandari, Agha A. Husain, Manveen S. Chadha, Ashish Gupta, Lossy and Lossless Current-mode Integrators using CMOS Current Mirrors, International Journal of Engineering Research and Development, 9(3), 2013, 34-41. 